\title{
Extended moment formation and magnetic ordering in the trigonal chain compound $\mathrm{Ca}_{3} \mathrm{Co}_{2} \mathrm{O}_{6}$
}

\author{
V. Eyert ${ }^{1 *}$, C. Laschinger ${ }^{1}$, T. Kopp ${ }^{1}$, R. Frésard ${ }^{2}$ \\ ${ }^{1}$ Institut für Physik, Universität Augsburg, 86135 Augsburg, Germany, \\ ${ }^{2}$ Laboratoire Crismat, UMR CNRS-ENSICAEN (ISMRA) 6508, Caen, France
}

\begin{abstract}
The results of electronic structure calculations for the one-dimensional magnetic chain compound $\mathrm{Ca}_{3} \mathrm{Co}_{2} \mathrm{O}_{6}$ are presented. The calculations are based on density functional theory and the local density approximation and used the augmented spherical wave (ASW) method. Our results allow for deeper understanding of recent experimental findings. In particular, alternation of Co $3 d$ low- and high-spin states along the characteristic chains is related to differences in the oxygen coordination at the inequivalent cobalt sites. Strong hybridization of the $d$ states with the O $2 p$ states lays ground for polarization of the latter and the formation of extended localized magnetic moments centered at the high-spin sites. In contrast, strong metal-metal overlap along the chains gives rise to intrachain ferromagnetic exchange coupling of the extended moments via the $d_{3 z^{2}-r^{2}}$ orbitals of the low-spin cobalt atoms.
\end{abstract}

Key words: density functional theory, low-dimensional compounds, magnetic chains, geometric frustration

PACS: 71.20.-b, 75.10.Pq, 75.30.Et

\section{Introduction}

Low-dimensional physical systems are attracting a lot of attention since long due to the occurence of exciting physical properties deviating distinctly from those known from three-dimensional systems. This includes both, new types of ordering phenomena being due to, e.g., Fermi-surface instabilities and new

\footnotetext{
* Corresponding author. fax: +49 8215983262

Email address: eyert@physik.uni-augsburg.de (V. Eyert $\left.{ }^{1}\right)$.
} 
types of excitations as resulting, e.g., from spin-charge separation. Furthermore, being embedded in three-dimensional crystals, low-dimensional atomic arrangements give rise to different length scales and coupling strengths and thus may lead to rich phase diagrams. Finally, the existence of magnetic moments in such systems raises questions concerning the relevant exchange mechanisms and their anisotropies.

In recent years, interest especially in one-dimensional systems has focused on a new class of transition metal oxides of the general formula $\mathrm{A}_{3}^{\prime} \mathrm{ABO}_{6}$ $\left(\mathrm{A}^{\prime}=\mathrm{Ca}, \mathrm{Sr}, \mathrm{Ba} ; \mathrm{A}, \mathrm{B}=\right.$ transition metal $)$, which crystallize in the trigonal $\mathrm{K}_{4} \mathrm{CdCl}_{6}$ structure [1]. The space group is $R \overline{3} c\left(D_{3 d}^{6}\right.$, No. 167). In these compounds, transition metal-oxygen polyhedra form well separated chains running parallel to the trigonal axis; see, e.g., Refs. [2,3] for a representation of the crystal structure. Space between the chains is filled with the $\mathrm{A}^{\prime}$ cations. Each chain consists of alternating, face-sharing $\mathrm{AO}_{6}$ trigonal prisms and $\mathrm{BO}_{6}$ octahedra. Typically, the ratio of interchain to intrachain metal-metal distance is of the order of two, this fact explaining the pronounced one-dimensionality. The chains themselves are arranged on a triangular lattice. As a consequence, in addition to showing the abovementioned unique properties these compounds allow to study geometric frustration effects as partial disorder and spin-glass like behaviour.

Prominent members of this class are the cobaltates $\mathrm{Ca}_{3} \mathrm{CoBO}_{6}$ with $\mathrm{B}=\mathrm{Co}$, $\mathrm{Rh}, \mathrm{Ir}$, which have gained much interest due to their exciting magnetic properties. Here we concentrate on $\mathrm{Ca}_{3} \mathrm{Co}_{2} \mathrm{O}_{6}$, which has two inequivalent cobalt sites, one in octahedral environment (labelled Co1) and the other (Co2) centered in the trigonal prisms [2,3]. According to powder neutron diffraction data reported by Fjellvåg et al. room-temperature lattice constants amount to $a_{\text {hex }}=9.0793 \AA$ and $c_{\text {hex }}=10.381 \AA[2]$. Data taken at $10 \mathrm{~K}$ by Aasland $e t$ al. resulted in $a_{\text {hex }}=9.060 \AA$ and $c_{\text {hex }}=10.366 \AA$ with only slight changes of the atomic positional parameters as compared to $298 \mathrm{~K}$ [3]. The latter data were used in the calculations.

While the Co-Co distance within the chains amounts to $2.595 \AA$, next-nearest neighbour distances across the chains are $5.31 \AA[2]$. This value is somewhat larger than the interchain separation of $5.24 \AA$, since neighbouring chains are shifted parallel to the trigonal axis by $1 / 3$ of the intrachain separation. According to the neutron diffraction data, the octahedral Co1-O distances of $1.916 \AA$ are considerably shorter than the $\mathrm{Co} 2-\mathrm{O}$ distances, which amount to $2.062 \AA[2]$. From the difference it was expected that $\mathrm{Ca}_{3} \mathrm{Co}_{2} \mathrm{O}_{6}$ might show charge or spin order at low temperatures [2]. Finally, the short Co1-Co2 distance within the chains, which is a result of the face-sharing of the polyhedra and is close to the value of $2.51 \AA$ in metallic cobalt, has been taken as indicative for strong metal-metal bonding [2]. 
Magnetic susceptibility data revealed the onset of magnetic ordering below $\mathrm{T}_{\mathrm{C} 1}=24 \mathrm{~K}[3,4,5]$. In addition, a second transition was found at $\mathrm{T}_{\mathrm{C} 2}=12 \mathrm{~K}$ $[4,5]$. From Curie-Weiss behaviour above $80 \mathrm{~K}$ an effective magnetic moment of $5.7 \mu_{B}$ and a paramagnetic Curie temperature of $28 \mathrm{~K}$ pointing to predominant ferromagnetic ordering was inferred. Neutron diffraction measurements by Aasland et al. revealed local magnetic moments of $0.08 \mu_{B}$ and $3.00 \mu_{B}$ for octahedral Co1 and trigonal prismatic Co2, respectively [3,4]. All moments are aligned along the trigonal axis. From these data it was furthermore concluded that the coupling is ferromagnetic within the chains and antiferromagnetic across the chains [3]. While $\mathrm{T}_{\mathrm{C} 1}$ has been associated with the ferromagnetic intrachain coupling, $\mathrm{T}_{\mathrm{C} 2}$ would correspond to long-range three-dimensional ordering $[4,5]$.

Magnetization measurements showed a plateau at $\approx 1.31 \mu_{B}$ per f.u. for low field and a steep increase by a factor of three at about $3.5 \mathrm{~T}$, which was interpreted as a ferri- to ferromagnetic transition [3,4]. However, the final magnetic moment of about $4 \mu_{B}$ was difficult to reconcile with the values found for the local cobalt moments $[3,6]$. While Aasland et al. proposed a magnetic structure with $1 / 3$ of the chains being antiparallel to the remaining $2 / 3$ [3], Kageyama et al. argued that at low field and temperatures between 10 and $24 \mathrm{~K}$ the system assumes a partially disordered antiferromagnetic (PDA) state [4]. The existence of this state in different materials is still a matter of controversal discussion. While it was also reported for $\mathrm{Ca}_{3} \mathrm{CoRhO}_{6}[7]$, recent magnetization and heat capacity data allowed to exclude the PDA state for $\mathrm{Ca}_{3} \mathrm{CoIrO}_{6}$ [8]. For $\mathrm{Ca}_{3} \mathrm{Co}_{2} \mathrm{O}_{6}$ it was shown that pressure leads to increase of $\mathrm{T}_{\mathrm{C} 2}$ and, hence, stabilization of the ferrimagnetic phase $[9,10]$. Magnetization data taken below $\mathrm{T}_{\mathrm{C} 2}$ on single crystals by Maignan et al. displayed a sequence of up to four different magnetization plateaus pointing to a variety of competing magnetic structures [5]. Moreover, AC- $\chi$ measurements by Maignan et al. showed a remarkably strong frequency shift, which has been interpreted as a measure of the frustration between different magnetic configurations [5]. These results supported interpretations of $\mathrm{Ca}_{3} \mathrm{Co}_{2} \mathrm{O}_{6}$ as a triangular lattice of antiferromagnetically coupled Ising spins, each formed from a single chain. However, the exact nature of the magnetic ground state of this material is still unclear.

In this letter we report on electronic structure calculations for $\mathrm{Ca}_{3} \mathrm{Co}_{2} \mathrm{O}_{6}$, which include the spin-degenerate case as well as spin-polarized configurations assuming both the spin-structure proposed by Aasland et al. and the hypothetical case of ferromagnetic alignment of the chains. Our calculations reveal i) low- and high-spin moments, respectively, at the octahedral and trigonal prismatic cobalt sites, ii) a rather large oxygen moment due to polarization by the high-spin cobalt sites, which together give rise to the formation of extended but still well localized $\mathrm{CoO}_{6}$ moments and resolve the discrepancy between the magnetization and neutron diffraction data, and iii) coupling of these extended moments by ferromagnetic exchange via the $3 d$ states of the 
low-spin octahedral cobalt atoms.

\section{Methodology}

The calculations were performed using the scalar-relativistic augmented spherical wave (ASW) method $[11,12]$. In order to represent the correct shape of the crystal potential in the large voids of the open crystal structure, additional augmentation spheres were inserted. Optimal augmentation sphere positions as well as radii of all spheres were automatically generated by the sphere geometry optimization (SGO) algorithm [13]. Self-consistency was achieved by an efficient algorithm for convergence acceleration [14]. Brillouin zone sampling was done using an increased number of k-points ranging from 28 to 408 points within the irreducible wedge.

\section{Results and Discussion}

\subsection{Spin-Degenerate Calculations}

In a first step the electronic properties of $\mathrm{Ca}_{3} \mathrm{Co}_{2} \mathrm{O}_{6}$ were calculated with spin-degeneracy enforced. The resulting electronic structure and partial densities of states (DOS) are displayed in Fig. 1. All bands display rather strong
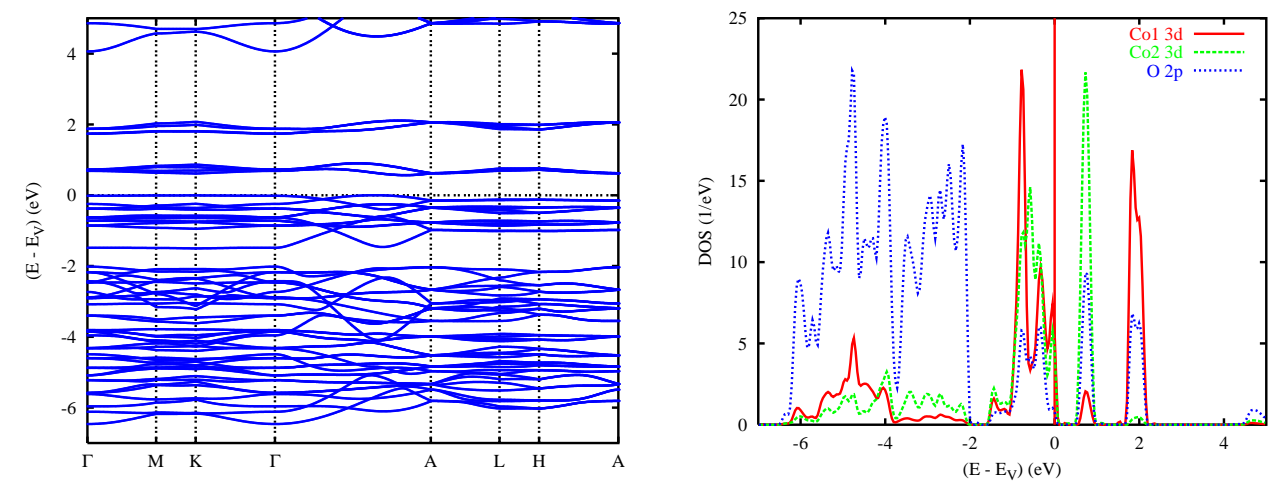

Fig. 1. Electronic bands and partial DOS of nonmagnetic $\mathrm{Ca}_{3} \mathrm{Co}_{2} \mathrm{O}_{6}$.

dispersion parallel to the chain axis, i.e. along the line $\Gamma$-A, reflecting the onedimensionality of the compound. However, the finite perpendicular dispersion points to albeit weak interchain coupling.

Five groups of bands are identified. In the energy range from -6.4 to - $2.0 \mathrm{eV} 36$ bands are observed, which trace back mainly to the $\mathrm{O} 2 p$ states. In contrast, 

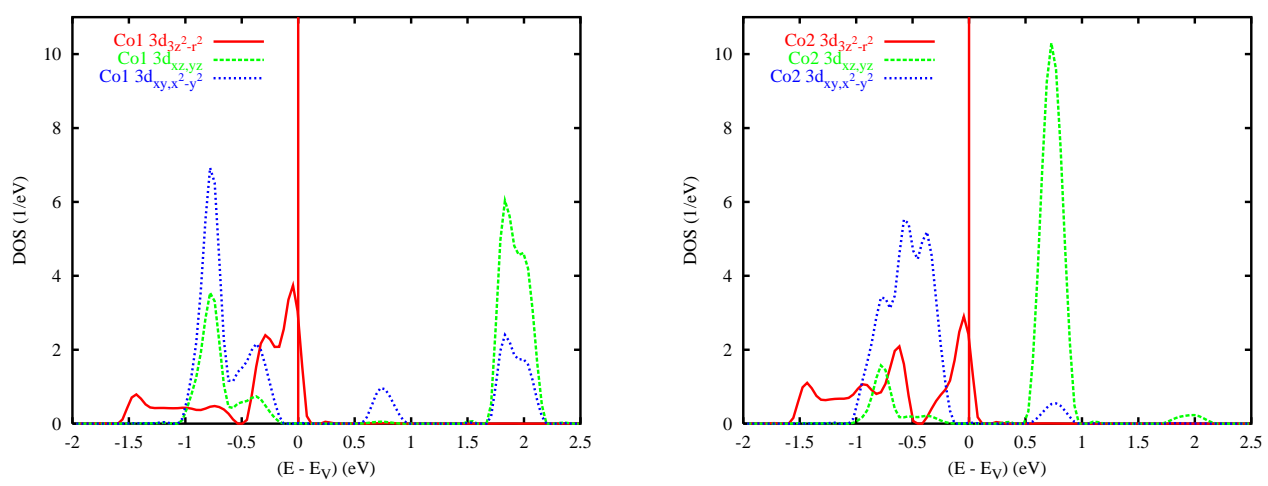

Fig. 2. Partial Co1 and $\mathrm{Co} 23 d$ DOS of nonmagnetic $\mathrm{Ca}_{3} \mathrm{Co}_{2} \mathrm{O}_{6}$. Slight broadening is due to the DOS calculation scheme [15].

the next three groups of bands, which extend from $-1.7 \mathrm{eV}$ to the valence band maximum, from 0.6 to $1.0 \mathrm{eV}$, and from 1.8 to $2.2 \mathrm{eV}$, comprise twelve and two times four bands, respectively, and derive mainly from the Co $3 d$ states. Finally, bands starting above $4 \mathrm{eV}$ are of $\mathrm{Co} 4 s$ character. As is obvious from the partial DOS, $p-d$ hybridization causes substantial $d$ and $p$ contributions, respectively, below and above $-2 \mathrm{eV}$ reaching about $40 \%$ especially in the upper valence band and the conduction bands. Whereas $d$ contributions from the octahedrally coordinated Co1 show up mainly below $-3.8 \mathrm{eV}$ and give rise to the peak at $-4.8 \mathrm{eV}$, the $\mathrm{Co} 2 d$ contributions are equally spread over the whole energy range between -6 and $-2 \mathrm{eV}$. In passing we mention the $d$ admixture to the peaks at 0.8 and $2.0 \mathrm{eV}$, which is of almost pure $\mathrm{Co} 2$ and $\mathrm{Co} 1$ character, respectively. From the partial DOS the cobalt atoms can be formally assigned a $d^{6}$ configuration, which, together with the absence of charge disproportionation, is in perfect agreement with the neutron scattering results [3].

Crystal field splitting due to the octahedral and trigonal prismatic environment of Co1 and Co2 atoms, respectively, is revealed on closer inspection of the partial $d$ DOS as displayed in Fig. 2. Note that we used for both sites the same global coordinate system with the $z$ axis parallel to the chain axis. It deviates from the natural coordinate system for octahedral coordination with the Cartesian axes parallel to the metal-oxygen bonds due to rotation of the octahedra centered at the Co1 sites. As a consequence, both the $t_{2 g}$ and $e_{g}$ bands arise as admixtures of all five $d$ states (referred to the global coordinate system), a situation well known from other trigonal and hexagonal systems as, e.g., $\mathrm{V}_{2} \mathrm{O}_{3}$ [16]. Nevertheless, at the $\mathrm{Co} 1$ sites, the octahedral crystal field leads to nearly perfect splitting into fully occupied $t_{2 g}$ and empty $e_{g}$ states. In contrast, the trigonal crystal field at the Co2 sites leads to splitting into non-degenerate $d_{3 z^{2}-r^{2}}$ as well as doubly degenerate $d_{x y, x^{2}-y^{2}}$ and $d_{x z, y z}$ states. While the latter appear almost exclusively in the peak around $0.75 \mathrm{eV}$, the former three orbitals dominate the upper valence band. 
Apart from the abovementioned strong $p$ - $d$ hybridization two findings are important for the understanding of the magnetic properties. First, as has already been mentioned, the lower and upper conduction band, respectively, centered at 0.8 and $2 \mathrm{eV}$ are due exclusively to the Co2 $d_{x z, y z}$ and Co1 $e_{g}$ states. This is related to the fact that the latter states experience $\sigma$-type $p$ - $d$ bonding and, being antibonding, are thus pushed to higher energies whereas the $p$ - $d$-bonding of the $d_{x z, y z}$ orbitals is less strong. In the present situation this difference lets us expect that in a spin-polarized calculation the Co2 sites will carry larger magnetic moments. Second, we point to the large width of the $d_{3 z^{2}-r^{2}}$ bands as obvious from both the Co1 and Co2 partial DOS, which is indicative of the strong metal-metal overlap along the chain axis. This has been confirmed by the calculated crystal orbital overlap population (COOP) and covalent bond energies as well as a more detailed analysis of the electronic wave functions. According to the latter the $d_{3 z^{2}-r^{2}}$ states give rise to the dispersionless bands along $\Gamma-\mathrm{M}-\mathrm{K}-\Gamma$ at $-1.5 \mathrm{eV}$ and at $\mathrm{E}_{\mathrm{F}}$ and show finite dispersion only parallel to $\Gamma$-A. Within these bands the bonding states are more Co2-like, whereas the antibonding states have increased Co1-like character.

\subsection{Spin-Polarized Calculations assuming Ferromagnetic Order}

In a second step, spin-polarized calculations were performed. Yet, in view of the predominant influence of the ferromagnetic interchain coupling as deduced from the experimental data we started out from the hypothetical situation, where all chains are aligned in parallel, before turning to the ferrimagnetic spin-structure proposed by Aasland et al. [3]. Insofar our procedure is along the same line of reasoning as the calculations by Whangbo et al., who, however, did not deal with the observed ferrimagnetic structure and concentrated mainly on the origin of the cobalt moments [17].

For the assumed ferromagnetic order we find a converged solution with well localized magnetic moments of $2.73,0.35$, and $0.14 \mu_{B}$, respectively, on Co2, Co1, and oxygen. These values reflect the experimental finding of a high and low spin state of the cobalt atoms at the trigonal prismatic and octahedral sites, respectively, and are close to those reported by Whangbo et al. as well as the neutron diffraction results of 3.00 and $0.08 \mu_{B}$ for the cobalt atoms. The total moment per unit cell, i.e. per two formula units, amounts to exactly $8.0 \mu_{B}$. Worth mentioning are the rather high magnetic moments associated with the oxygen sites. Summing up to almost $1 \mu_{B}$ per formula unit, they pro-

vide an easy explanation for the discrepancy between the neutron scattering data and the magnetization in high field.

The Co $3 d$ partial DOS given in Fig. 3 display a rather complex relationship between the electronic and magnetic properties. Due to the reduced magnetic 

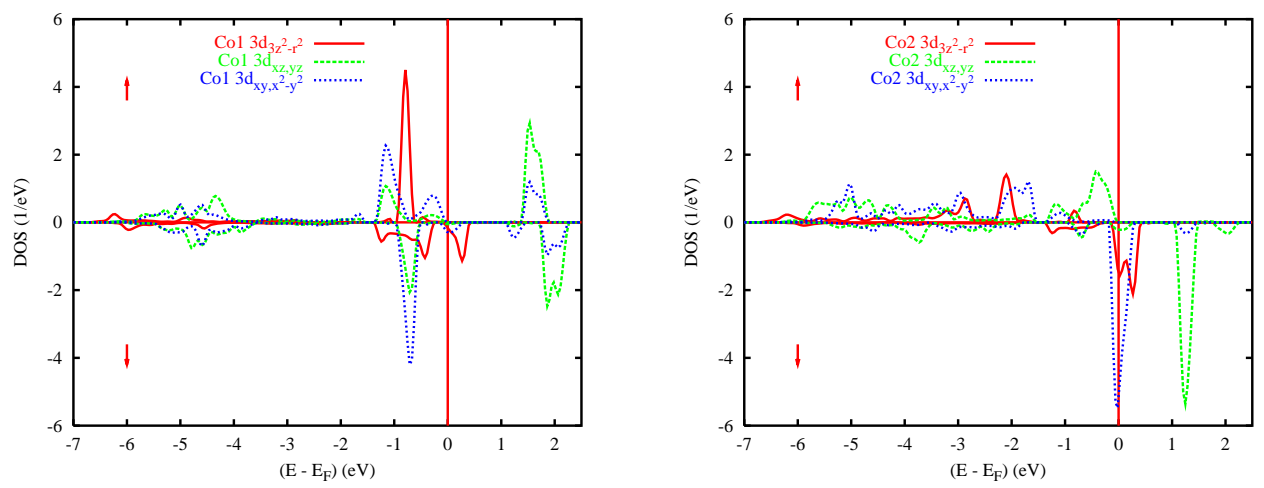

Fig. 3. Partial Co1 and Co2 3d DOS of assumed ferromagnetic $\mathrm{Ca}_{3} \mathrm{Co}_{2} \mathrm{O}_{6}$.
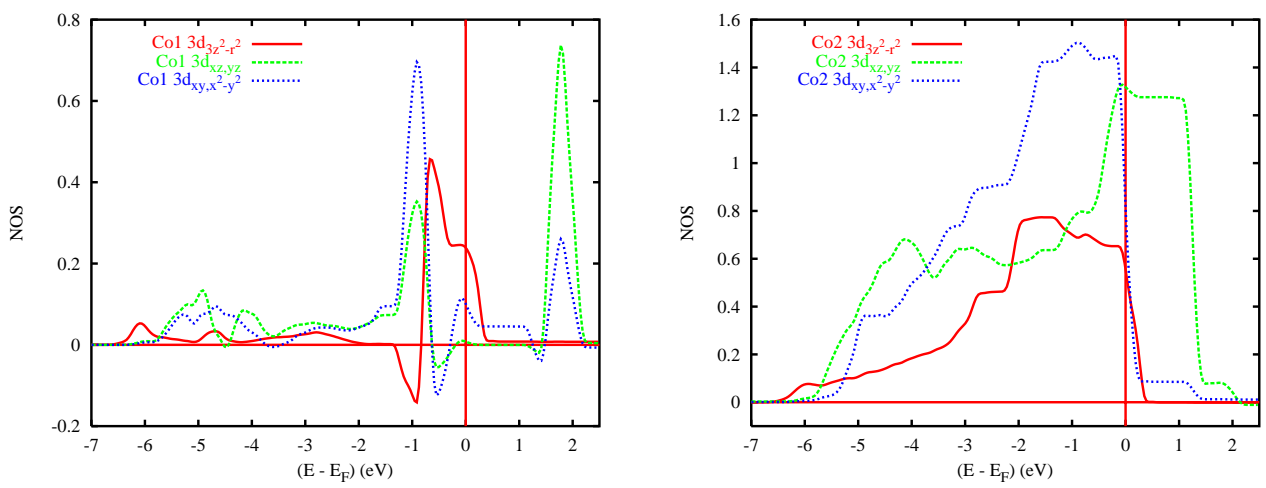

Fig. 4. Partial Co1 and Co2 $3 d$ integrated moments of assumed ferromagnetic $\mathrm{Ca}_{3} \mathrm{Co}_{2} \mathrm{O}_{6}$.

moment the Co1 $3 d$ states show only small spin splitting of the order of $0.3 \mathrm{eV}$, hence, much smaller than the crystal field splitting. As a consequence, the magnetic moment is carried alone by the $t_{2 g}$ states. Within this group of bands spin-majority $d_{3 z^{2}-r^{2}}$ states experience a pronounced localization, whereas the spin-minority states have a much larger band width. For the remaining $t_{2 g}$ states things are reversed. Co1 $d$ contributions below $-3.5 \mathrm{eV}$ display small spin-splitting but give only a negligible contribution to the magnetic moment. This is related to the fact that $d-p$ overlap within the octahedra is strongest for states of $e_{g}$ symmetry, which are completely occupied or empty. The situation is different at the trigonal prismatic sites, where the spin-splitting is of the same order of magnitude as the crystal field splittings. As a consequence, spin-majority states are shifted to the energy region, where the oxygen $2 p$ states dominate. At the same time, these states experience considerable delocalization. In contrast, spin-minority states form sharp peaks at and above the Fermi energy but give only minor contributions to the occupied states.

The different distributions of the electronic states in the octahedra and trigonal prisms have interesting consequences for the magnetic moments. This becomes clearer from Fig. 4, which displays energy and orbital-dependent spin 
magnetizations, i.e. the integrated differences of the spin-dependent partial DOS. For the Co1 sites it is easily seen that the small magnetic moment is mainly carried by the $d_{3 z^{2}-r^{2}}$ states. It starts to build up in the energy region above $-1.5 \mathrm{eV}$ and is thus due to orbitals well localized within the Co1 atoms. This is completely different for the trigonal prismatic sites. There the magnetic moment is rather isotropic, i.e. it grows out of similar amounts from all five $d$ states. According to Fig. 4 the $d_{3 z^{2}-r^{2}}$ orbitals contribute $0.6 \mu_{B}$, while the doubly degenerate $d_{x z, y z}$ and $d_{x^{2}-y^{2}, x y}$ states give $1.3 \mu_{B}$ and $0.8 \mu_{B}$, respectively. Furthermore, as is clearly seen in Fig. 4, all these moments start to build up already well within the energy region, where the oxygen states are dominating, thus reflecting the considerable oxygen polarization. To conclude, the strong $p$ - $d$ hybridization within the trigonal prisms, which has a strength between the rather nonbonding $\pi$-type and the directed $\sigma$-type overlap of the octahedra, leads to the formation of a combined isotropic $\mathrm{Co} 2 d$-O $p$ magnetic moment. Note that the oxygen $p$ orbitals participate in the local moments and not in the exchange interaction between the prisms. This situation goes under the name extended moment formation and has been previously observed in copper oxides $[18,19]$. Finally, coupling between these extended localized moments is mediated by ferromagnetic exchange interaction via the $d_{3 z^{2}-r^{2}}$ orbitals of the low-spin Co1 atoms.

\subsection{Spin-Polarized Calculations with Ferrimagnetic Order}

In a last step, we turn to the ferrimagnetic structure, where $1 / 3$ of the chains have their spins antiparallel to the remaining chains. The results obtained from these calculations are very similar to those for the hypothetical ferromagnetic structure and, hence, provide an a posteriori justification for the validity of the latter. Local magnetic moments of $2.72,0.34$, and $0.14 \mu_{B}$, respectively, are found for Co2, Co1, and oxygen and the total moment per unit cell again amounts to $\approx 8.00 \mu_{B}$. According to the calculated magnetic moments as well as the partial DOS, which likewise look very similar to those obtained for the ferromagnetic situation, the differences between the spin up and down chains are rather small. Yet, slightly sharper peaks, i.e. stronger localization of the Co $3 d$ states, are observed for the minority chains. The general situation obtained for the ferrimagnetic structure is thus very similar to that grown out of the calculations for ferromagnetically coupled chains.

\section{Conclusion}

In conclusion, electronic structure calculations for the magnetic chain compound $\mathrm{Ca}_{3} \mathrm{Co}_{2} \mathrm{O}_{6}$ have revealed strong influence of the local coordination 
polyhedra on the electronic and magnetic properties. In particular, shorter bond lengths and strong $\sigma$-type bonding in the octahedra allow for only small spin-splitting at these sites. In contrast, due to smaller crystal field splittings in trigonal prismatic surrounding the Co2 atoms are found in a high-spin state. Strong $d-p$ hybridization causes rather large oxygen magnetic moments, which, together with the cobalt moments at the trigonal prismatic sites, take part in the formation of extended but still well localized magnetic moments. Moreover, intrachain exchange coupling between these effective moments is based on metal-metal overlap of the cobalt $d_{3 z^{2}-r^{2}}$ orbitals along the chains and mediated by ferromagnetic exchange interaction via the $d_{3 z^{2}-r^{2}}$ orbitals of the low-spin cobalt atoms. Recently, mapping these results onto a Heisenberg model we were able to underline the importance of the interplay of different crystal field splittings, $d-p$ hybridizations, and metal-metal overlap for the fer-

romagnetic intrachain order [20]. Finally, according to a detailed analysis of the oxygen magnetic moments as well as the analysis within the Heisenberg model the interchain coupling seems to grow out of super-superexchange via oxygen states.

\section{Acknowledgments}

We are indebted to D. Khomskii and A. Maignan for fruitful discussions. C. Laschinger gratefully acknowledges a Marie Curie fellowship of the European Community program under number HPMT2000-141. This work was supported by the Deutsche Forschungsgemeinschaft (DFG) through Sonderforschungsbereich SFB 484 and by the BMBF (13N6918A).

\section{References}

[1] K. E. Stitzer, J. Darriet, and H.-C. zur Loye, Curr. Opin. Solid State Mater. Sci. 5 (2001) 535.

[2] H. Fjellvåg, E. Gulbrandsen, S. Aasland, A. Olsen, and B. C. Hauback, J. Solid State Chem. 124, (1996) 190.

[3] S. Aasland, H. Fjellvåg, and B. C. Hauback, Solid State Comm. 101, (1997) 187.

[4] H. Kageyama, K. Yoshimura, K. Kosuge, H. Mitamura, and T. Goto, J. Phys. Soc. Japan 66, (1997) 1607.

[5] A. Maignan, C. Michel, A. C. Masset, C. Martin, and B. Raveau, Eur. Phys. J. B 15, (2000) 657. 
[6] H. Kageyama, K. Yoshimura, K. Kosuge, M. Azuma, M. Takano, H. Mitamura, and T. Goto, J. Phys. Soc. Japan 66, (1997) 3996.

[7] S. Niitaka, K. Yoshimura, K. Kosuge, M. Nishi, and K. Kakurai, Phys. Rev. Lett. 87, (2001) 177202.

[8] S. Rayaprol, K. Sengupta, and E. V. Sampathkumaran, Phys. Rev. B 67, (2003) 180404.

[9] B. Martínez, V. Laukhin, M. Hernando, J. Fontcuberta, M. Parras, and J. M. González-Calbet, Phys. Rev. B 64, (2001) 012417.

[10] M. Hernando, B. Martínez, V. Laukhin, J. Fontcuberta, M. Parras, and J. M. González-Calbet, J. Magn. Magn. Mat. 242-245, (2002) 757.

[11] A. R. Williams, J. Kübler, and C. D. Gelatt, Jr., Phys. Rev. B 19, (1979) 6094.

[12] V. Eyert, Int. J. Quantum Chem. 77, (2000) 1007.

[13] V. Eyert and K.-H. Höck, Phys. Rev. B 57, (1998) 12727.

[14] V. Eyert, J. Comp. Phys. 124, (1996) 271.

[15] M. Methfessel and A. T. Paxton, Phys. Rev. B 40, (1989) 3616.

[16] K. Held, G. Keller, V. Eyert, D. Vollhardt, and V. I. Anisimov, Phys. Rev. Lett. 86 (2001) 5345.

[17] M.-H. Whangbo, D. Dai, H.-J. Koo, and S. Jobic, Solid State Comm. 125, (2003) 413.

[18] V. Eyert, K.-H. Höck, and P. S. Riseborough, Europhys. Lett. 31, (1995) 385.

[19] R. Weht and W. E. Pickett, Phys. Rev. Lett. 81, (1998) 2502.

[20] R. Frésard, C. Laschinger, T. Kopp, and V. Eyert, (cond-mat/0309031). 